\title{
Multisite spectroscopic and photometric observations of the pulsating sdB star PG 1605+072
}

\author{
S.J. O'Toole ${ }^{1}$, S. Falter ${ }^{1}$, U. Heber ${ }^{1}$, C.S. Jeffery ${ }^{2}$, S. Dreizler ${ }^{3,4}$, \\ S.L. Schuh ${ }^{3,4}$, the $\mathrm{MSST}^{5}$ and $\mathrm{WET}^{6}$ teams \\ ${ }^{1}$ Dr Remeis-Sternwarte, Astronomisches Institut der Universität \\ Erlangen-Nürnberg, Sternwartstr. 7, Bamberg D-96049, Germany \\ ${ }^{2}$ Armagh Observatory, College Hill, Armagh BT61 9DG, Northern \\ Ireland, UK \\ ${ }^{3}$ Insitut für Astronomie und Astrophysik, Universität Tübingen, Sand 1, \\ D-72076 Tübingen, Germany \\ ${ }^{4}$ Universitätssternwarte, Universität Göttingen, Geismarlandstrasse 11, \\ D-37083 Göttingen, Germany
}

\begin{abstract}
We present the first results from the MultiSite Spectroscopic Telescope (MSST) observations of the sdBV star PG 1605+072. Pulsating sdB stars (V361 Hya stars) offer the chance to gain new insights into the formation and evolution of extreme Horizontal Branch stars using the tools of asteroseismology. PG $1605+072$ is an outstanding object in its class, with the richest frequency spectrum, the longest periods, and the largest variations. The MSST campaign took place in 2002 May/June and we present here the massive data set, made up of $399 \mathrm{hr}$ of photometry and $151 \mathrm{hr}$ of spectroscopy. The overall aims of the project are to examine light/velocity amplitude ratios and phase differences, changes in equivalent width/line index, and $\lambda$-dependence of photometric amplitudes, and to use these properties for mode identification.
\end{abstract}

\section{Motivation}

With the highest amplitudes and longest periods of any known sdBV star (officially known as V361 Hya stars), PG $1605+072$ is an ideal target for timeresolved spectroscopic observations. Intensity variations were first detected by Koen et al. (1998); subsequent multisite observations by Kilkenny et al. (1999) found around 50 pulsation frequencies. Heber et al. (1999) showed that the star has $v \sin i=39 \mathrm{~km} \mathrm{~s}^{-1}$, which will further complicate the frequency spectrum. Using 2-m telescopes and low resolution spectra, O'Toole et al. (2000) detected velocity variations in the Balmer lines with amplitudes up to $\sim 14 \mathrm{~km} \mathrm{~s}^{-1}$. Woolf et al. (2002) and Falter et al. (2003) showed that using 4-m telescopes with higher resolution significantly improved the velocity accuracy, with the former

\footnotetext{
${ }^{5}$ http://astro . uni-tuebingen. de/ schuh/msst/astronomers . html

${ }^{6}$ http: //wet.iitap.iastate.edu/xcov22/people.html
} 


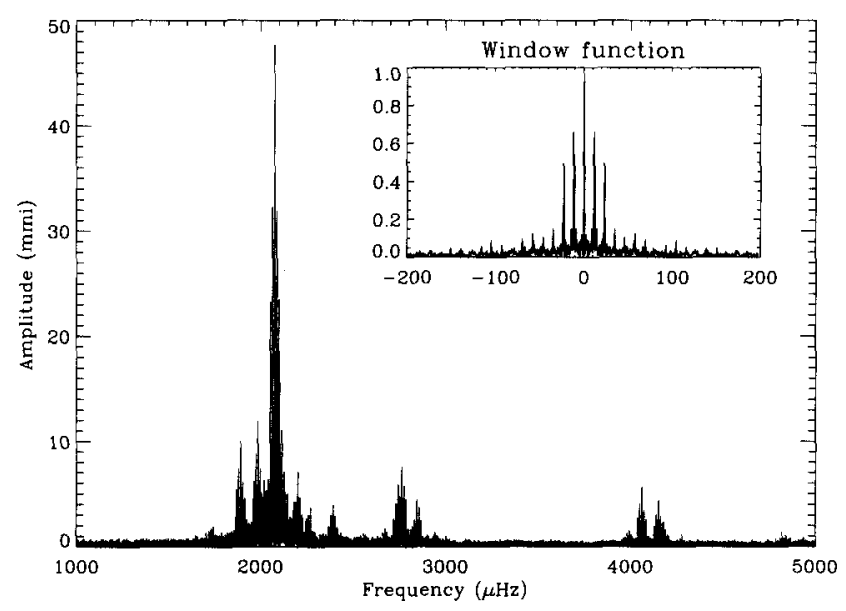

Figure 1. Amplitude spectrum of MSST photometry from SAAO, SSO and the JKT. The $2075.8 \mu \mathrm{Hz}$ mode is dominant again. The inset shows the spectral window.

study finding evidence for distortions in the Balmer line profiles. O'Toole et al. (2003) measured Balmer line indices and derived the effective temperature and surface gravity variations for eight of the pulsation frequencies.

These analyses showed, however, that one and two site spectroscopic campaigns are not enough to understand the complex nature of PG 1605+072, and that even multisite photometry could not do the job alone. Considering the potentially huge amount of information that can be obtained from this star, we decided to undertake a multisite spectroscopic and photometric campaign in 2002 May/June. Here we present the initial results from this ambitious project.

\section{Observations and reductions}

There were two sets of photometric observations of PG 1605+072. Immediately prior to the main MSST campaign, the star was observed as an alternate target in the WET Xcov22 run. Further details can be found in Schuh et al. (2003). There were also two parts to the spectroscopic campaign, separated by about two weeks. Details of observations from the main part of the MSST campaign can be found in Heber et al. (2003).

The photometric observations were reduced using the WET package qed for the photomultiplier data, while IRAF and TRIPP (see Schuh et al. 2003) were used for the CCD data. The 2-m spectroscopic observations were all reduced using IRAF, including a double precision version of the rv package ${ }^{1}$, while the

${ }^{1}$ available from http://iraf.noao.edu/scripts/extern/rvx.pl 


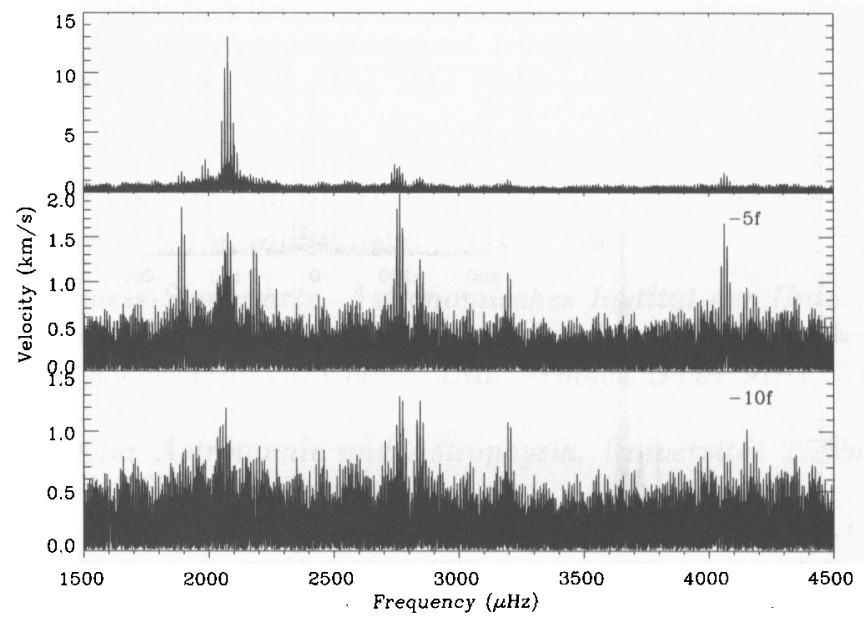

Figure 2. Velocity amplitude spectrum of PG 1605+072 (top) from 2-m spectroscopy; after prewhitening 5 frequencies (middle); after prewhitening by 10 frequencies (bottom).

4-m data were reduced using SPEX (long-slit SPectrum EXtraction package ${ }^{2}$ ). All of the 4-m spectra were trailed (i.e. the star was moved down the slit at a constant rate), and SPEX contains routines designed to reduce this kind of spectrum.

\section{Preliminary results}

The project is beginning to produce some results, although it has been slowed down by some timing-related problems in the photometry. The exact causes for these are unknown, and further work is needed to determine how to correct them. The amplitude spectrum of data from the three sites we are most confident with is shown in Fig. 1. The dominant mode is at $2075.8 \mu \mathrm{Hz}$, as seen in the most recent study of Falter et al. (2003). This mode had a much lower amplitude in the velocity studies of O'Toole et al. (2000, 2002) and Woolf et al. (2002). This effect is also seen in the 2-m spectroscopy (Fig. 2). Currently, the 4-m spectroscopy has been reduced, but not completely analysed. A sample of the velocity curve from the Calar Alto 3.5-m telescope is shown in Fig. 3.

\section{Conclusions and the future}

There is still plenty of work to do before a proper and detailed analysis of the MSST observations can be done. Reduction of the photometry and analysis of

\footnotetext{
${ }^{2}$ see http://astro.uni-tuebingen.de/ $\sim$ schuh/spex/index.html
} 


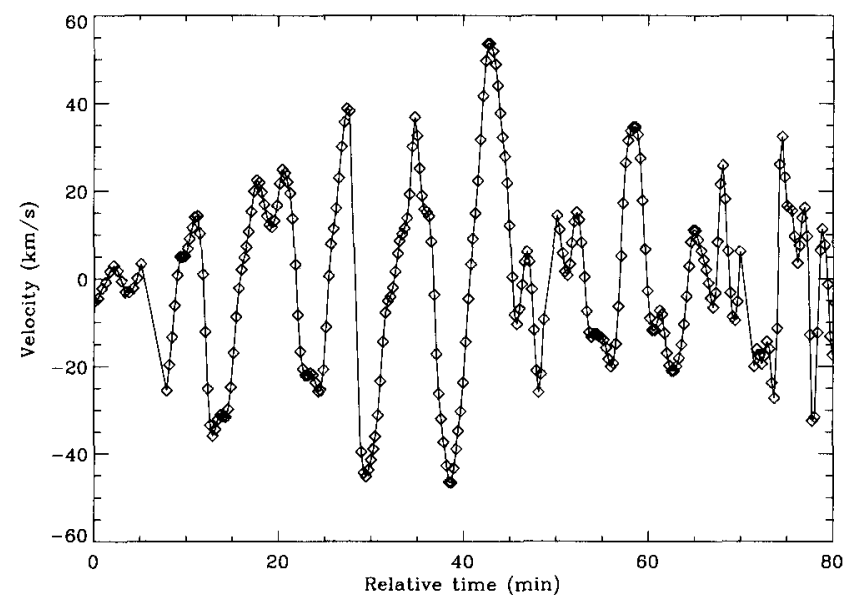

Figure 3. Velocity curve from the Calar Alto 3.5-m telescope with the TWIN spectrograph. Variations with a period of $\sim 8 \mathrm{~min}$ are clear.

the 4-m spectroscopy needs to be completed. The timing problems need to be investigated and then corrected for, after which combination of all photometric (MSST+WET) data can be done. Only then can frequencies, amplitudes and phases from photometry and spectroscopy be compared, and the identification of modes in PG 1605+072 begin in earnest. We will call on the pulsation theorists to help explain some of our results.

\section{References}

Falter, S., Heber, U., Dreizler, S., et al. 2003 A\&A, 401, 289

Heber, U., et al. 2003, 13th European Workshop on White Dwarfs. NATO-ARW Workshop Series, p. 105

Kilkenny, D., Koen, C., O'Donoghue, D., et al. 1999 MNRAS, 303, 525

Koen, C., O'Donoghue, D., Kilkenny, D., et al. 1998 MNRAS, 296, 317

O'Toole, S.J., Bedding, T.R., Kjeldsen, H., et al. 2000 ApJ, 537, L53

O'Toole, S.J., Bedding, T.R., Kjeldsen, H., et al. 2002 MNRAS, 334, 471

O'Toole, S.J., Jørgensen, M.A.S.G., Kjeldsen, H., et al. 2003 MNRAS, 340, 856

Schuh, S.L., Dreizler, S., Deetjen, J.L., Göhler, E. 2003, Baltic Astron., 12, 167

Woolf, V.M., Jeffery, C.S., Pollacco, D. 2002, MNRAS, 339, 497 